\title{
Correction to: Geometric Properties of the Products of Modified Bessel Functions of the First Kind
}

\author{
Khaled Mehrez ${ }^{1,2}$ (D) Sourav $\operatorname{Das}^{3}$ (D) Anish Kumar ${ }^{3}$
}

Published online: 26 April 2021

(c) Malaysian Mathematical Sciences Society and Penerbit Universiti Sains Malaysia 2021

\section{Correction to: Bull. Malays. Math. Sci. Soc. https://doi.org/10.1007/s40840-021-01082-2}

In the initial online publication there was an error in Lemma 2.8. The original article has been corrected.

The changes below were made to the original article.

- Lemma 2.8 was removed from the article.

- Theorem 4.3 now is written as follows:

Theorem 4.3 Assume that the hypotheses $\left(H_{1}\right)$ of Theorem 3.1 are satisfied; in addition, assume that

$$
\left(H_{3}\right): 2 u v \geq(m+3)+\sqrt{(m+1)(19 m+49)} .
$$

Or, we suppose that the following conditions holds true:

$$
\left(H_{3}^{1}\right): u, v>0 ; \quad u v>2(e-1)(u+v)+(4 e-3) .
$$

The original article can be found online at https://doi.org/10.1007/s40840-021-01082-2.

Khaled Mehrez

k.mehrez@yahoo.fr

Sourav Das

souravdasmath@gmail.com; souravdas.math@nitjsr.ac.in

Anish Kumar

ak8107690@gmail.com

1 Department of Mathematics, Faculty of Sciences of Tunis, University of Tunis El Manar, 1060 Tunis, Tunisia

2 Department of Mathematics, Kairouan Preparatory Institute For Engineering Studies, University of Kairouan, 3100 Kairouan, Tunisia

3 Department of Mathematics, National Institute of Technology Jamshedpur, Jamshedpur, Jharkhand 831014, India 
Then the function $\mathcal{I}_{u, v}(z)$ is starlike in $\mathcal{D}$.

- In the proof of Theorem 4.3, the following sentence was removed:

Next, we suppose that the second assumptions hold true, the proof of this result goes along the lines introduced in Theorem 4.1 when we used Lemma 2.8, and we omit the details.

- Next, Corollary 4.4 was changed to:

Corollary 4.4 If $u \geq 7.88$, then the function $\mathcal{J}_{u}(z)$ defined in (1.4) is starlike in $\mathcal{D}$.

- In the proof of this corollary, the following lines were removed:

On setting $u=v$ in Theorem 4.3, second assumptions, we obtain that the function $\mathcal{J}_{u}(z)$ defined in (1.4) is starlike in $\mathcal{D}$ under the conditions

$$
2 u^{3}+(8-2 \sqrt{5}) u^{2}+(10-4 \sqrt{5}-2 e \sqrt{5}) u+4-\sqrt{5}(1+3 e)>0,
$$

which holds true for all $u \geq 2.28$.

\section{References}

1. Mehrez, K., Das, S., Kumar, A.: Geometric properties of the products of modified Bessel functions of the first kind. Bull. Malays. Math. Sci. Soc. (2021). https://doi.org/10.1007/s40840-021-01082-2

Publisher's Note Springer Nature remains neutral with regard to jurisdictional claims in published maps and institutional affiliations. 